\title{
Multilevel percutaneous kyphoplasty in painful osteolytic vertebral metastases: a study of the efficacy and safety
}

This article was published in the following Dove Press journal:

Journal of Pain Research

\section{Guan Shi \\ Fei Feng \\ Hao Chen \\ $\mathrm{Pu}$ Jia \\ Li Bao \\ Hai Tang}

Department of Orthopaedics, Beijing Friendship Hospital, Capital Medical University, Beijing, People's Republic of China
Correspondence: Hai Tang

Department of Orthopaedics, Beijing Friendship Hospital, Capital Medical

University, No. 95, Yong An Rd, XiCheng

District, Beijing 100050, People's Republic of China

Tel +86I360 I362187

Email tanghai2187@I26.com
Purpose: The spine is the most common skeletal site for metastatic tumors. In the treatment of vertebral metastases, the absolutely safe number of levels that can be treated via percutaneous kyphoplasty (PKP) during one procedure remains controversial. Thus, the present study aimed to evaluate the safety and efficacy of multilevel (>3) PKP for painful osteolytic vertebral metastases.

Patients and methods: We retrospectively analyzed the data from 176 patients who received PKP for painful osteolytic spinal metastases. Group A $(n=104)$ received PKP at a maximum of three vertebral levels per procedure, while group $B(n=72)$ received PKP at more than three levels during one operation. Surgical efficacy was assessed via a comparison of the VAS, Oswestry Disability Index (ODI), and general health (GH) and mental health (MH) scores of the Short Form-36 Health Survey before and after PKP. The complications were observed to evaluate the safety.

Results: Both groups had significantly improved VAS, ODI, GH and MH scores after PKP $(P<0.05)$. One week after surgery, group A had significantly less pain (VAS 3.41 \pm 0.1$)$ than group B (VAS 3.74 \pm 0.13$)(P<0.05)$. At 3 and 6 months postoperatively, the GH score was more significantly improved in group A than group B $(P<0.05)$. There were no significant differences between the two groups in the ODI, MH score, and complications $(P>0.05)$.

Conclusion: Multilevel PKP is safe and results in effective pain relief, and improvement of spinal mobility and GH in patients with osteolytic vertebral metastases. However, patients who undergo PKP at more than three levels have slightly worse short-term pain relief (less than 1 week postoperatively) and improvement of GH in the long-term (more than 3 months postoperatively) compared with patients who undergo PKP at less than three levels.

Keywords: multilevel, percutaneous kyphoplasty, osteolytic, vertebral metastases

\section{Introduction}

The spine is the most vulnerable skeletal system site for metastatic tumors. ${ }^{1}$ Osteolytic vertebral metastases may cause chronic and severe pain that significantly decreases quality of life. ${ }^{2}$ For patients with vertebral metastases without intraspinal tumorous masses, one of the best palliative surgical treatments is percutaneous kyphoplasty (PKP). PKP achieves satisfactory clinical results via the injection of bone cement to stabilize the pathological fractures and quickly relieve pain. ${ }^{3-5}$ Due to the potential for complications such as bone cement leakage and venous embolism, most clinicians tend to perform PKP at a maximum of three levels during each surgery. ${ }^{5,6}$ However, 
for patients with multilevel $(>3)$ vertebral metastases, the requirement for repeat PKP increases the medical costs and psychological burden. Therefore, an increasing number of clinicians are performing multilevel $(>3)$ PKP to treat vertebral metastases. $^{7-9}$ Data on the safety and efficacy of multilevel PKP for osteolytic vertebral metastases is currently very limited. Therefore, the present retrospective study was designed to evaluate the safety and efficacy of multilevel $(>3)$ PKP for painful osteolytic vertebral metastases.

\section{Patients and methods}

All patients were informed of their inclusion in the present study. Furthermore, all patients were informed about the possible benefits and complications of the technique itself, and provided written consent for both the procedure and for study inclusion. The study received approval from the review board of our institution.

\section{Patient selection and evaluation}

Between January 2010 and January 2016, PKP was performed in 176 patients with painful osteolytic spinal metastases. The study population comprised 88 males and 88 females, with an average age of 66.85 \pm 1.07 years (range 47 to 85 years) (Table 1). The patients were divided into two groups in accordance with the number of levels at which PKP was performed during the one surgery. Group A received PKP at a maximum of three levels $(n=104,59.09 \%)$, while group $\mathrm{B}$ received PKP at more than three levels $(\mathrm{n}=72,40.91 \%)$. In group A, PKP was performed at one level in 33 patients, two levels in 44 patients, and three levels in 35 patients. In group B, PKP was performed at four levels in 26 patients, five levels in 24 patients, six levels in 19 patients, and seven levels in five patients. A total of 564 vertebral bodies were treated with PKP. In group A, PKP was performed in 98 thoracic vertebrae (17.38\%), 100 lumbar vertebrae $(17.73 \%)$, and six sacral vertebrae (1.06\%). In group B, PKP was performed in 214 thoracic vertebrae (37.94\%), 134 lumbar vertebrae (23.76\%), and 12 sacral vertebrae $(2.13 \%)$. The three most common primary tumor sites were the lung (40.91\%), breast (16.48\%), and esophagus (9.66\%).

The patients were selected in accordance with the selection criteria (Table 2).
Table I Baseline characteristics of the two groups

\begin{tabular}{|l|l|l|l|}
\hline Characteristics & $\begin{array}{l}\text { Group } \\
\text { A (n=104) }\end{array}$ & $\begin{array}{l}\text { Group } \\
\text { B (n=72) }\end{array}$ & P-value \\
\hline Age (years) & $66.62 \pm 1.437$ & $67.19 \pm 1.615$ & 0.792 \\
Female/male & $44 / 60$ & $44 / 28$ & \\
Thoracic vertebrae & $98(48.04 \%)$ & $214(59.44 \%)$ & \\
Lumbar vertebrae & $100(49.02 \%)$ & $134(37.22 \%)$ & \\
Sacral vertebrae & $6(2.94 \%)$ & $12(3.33 \%)$ & \\
Revised Tokuhashi & $7.62 \pm 0.09$ & $7.35 \pm 0.11$ & 0.06 \\
Primary tumor & & & \\
Nasopharyngeal & 1 & 0 & \\
carcinoma & & & \\
Gallbladder cancer & 1 & 0 & \\
Lung cancer & 44 & 28 & \\
Liver cancer & 1 & 0 & \\
Melanoma & 2 & 0 & \\
Thyroid cancer & 3 & 1 & \\
Lymphoma & 1 & 0 & \\
Prostate cancer & 6 & 3 & \\
Breast cancer & 17 & 12 & \\
Soft palate cancer & 1 & 0 & \\
Tongue cancer & 4 & 1 & \\
Kidney cancer & 8 & 2 & \\
Esophagus cancer & 7 & 2 & \\
Gastric cancer & 2 & 10 & \\
Small bowel cancer & 1 & 2 & \\
Thymic cancer & 1 & & \\
Pancreatic cancer & 2 & & \\
Rectal cancer & 2 & & \\
Ovarian cancer & 3 & & \\
\hline
\end{tabular}

Table 2 Patient selection criteria

\begin{tabular}{|l|l|}
\hline Inclusion criteria & Exclusion criteria \\
\hline $\begin{array}{l}\text { Patients were diagnosed with } \\
\text { magnetic resonance imaging or } \\
\text { bone scan }\end{array}$ & $\begin{array}{l}\text { Patients received surgical treat- } \\
\text { ments including total tumor } \\
\text { resection, vertebral body repla- } \\
\text { cement and fixateur interne }\end{array}$ \\
\hline $\begin{array}{l}\text { Patients suffered severe back } \\
\text { pain and could not be relieved by } \\
\text { drugs }\end{array}$ & $\begin{array}{l}\text { Patients suffered severe coagu- } \\
\text { lopathy and severe infections }\end{array}$ \\
\hline $\begin{array}{l}\text { Patients' survival time was more } \\
\text { than } 3 \text { months }\end{array}$ & $\begin{array}{l}\text { Patients suffered paraparesis/ } \\
\text { paraplegia due to intraspinal } \\
\text { tumor masses that required } \\
\text { urgent decompression surgery }\end{array}$ \\
\hline
\end{tabular}

\section{Data collection}

\section{Efficacy of percutaneous kyphoplasty}

The VAS, Oswestry Disability Index (ODI), and the general health $(\mathrm{GH})$ and mental health $(\mathrm{MH})$ scores of the Short Form- 
36 Health Survey were assessed before and after PKP to evaluate the clinical efficacy.

The VAS is a subjective measurement of pain, where a score of 0 represents no pain, while a score of 10 represents the most severe pain imaginable.

The ODI is a subjective measure of functional disability. An ODI of 0 indicates normal functional ability. The more severe the patient's spinal mobility dysfunction is, the closer the ODI is to $100 \%$.

The GH score measures the individual's assessment of their own health and development, with higher scores indicating better GH. The MH score is a subjective measure of four types of psychological feelings (motivation, depression, behavior, and emotionally out of control); higher $\mathrm{MH}$ scores indicate better $\mathrm{MH}$.

\section{Safety}

Computed tomography was performed at 3 days postoperatively to detect bone cement leakage, including leakage into the intervertebral disc, the intraspinal region, and the paravertebral region. Patients were monitored for complications such as pulmonary embolism, discitis, infection, nerve injury, and bone cement syndrome during the follow-up period.

\section{Percutaneous kyphoplasty procedure}

All PKP procedures were performed by Dr. Tang, who had completed nearly 10,000 vertebral augmentation procedures.

The patient was in the prone position, and the heart rate, blood pressure, and pulse oxygenation were monitored throughout the procedure. C-arm fluoroscopy was used to confirm the diseased vertebrae, and local anesthesia ( $2 \%$ lidocaine and ropivacaine in a 1:1 ratio) was administered after disinfection. Under fluoroscopy, the needle with a detachable needle seat was inserted into the collapsed vertebral body through the pedicle. After confirmation that the needle was in the correct location, the needle holder was removed. The working sleeve was placed along the needle core, and then the needle core was removed to establish a working channel. A biopsy was taken through a hollow vertebral body drill. The vertebral body drill was removed after the required depth was reached. The balloon was delivered into the collapsed vertebral body. Fluoroscopy was used to confirm that the two marking rings of the balloon completely protruded from the working cannula. A gauge pressurizer (maximum pressure 20 ATM) was used to slowly inject the contrast agent to expand the balloon and form a cavity in the collapsed vertebral body. Subsequently, the pressure and volume were calculated, and the contrast agent was aspirated to make the balloon retract after the vacuum was slowly withdrawn. Under fluoroscopy, polymethylmethacrylate (PMMA) (Osteopal V, Heraeus Medical, Germany) was injected into the vertebral body. PMMA was injected at the late stage when it had a relatively thick consistency. During the PMMA injection, the distribution of bone cement in the vertebral body was closely observed. Once the bone cement neared the posterior wall of the vertebral body or the paravertebral venous plexus, the injection was stopped immediately to prevent the bone cement from entering the spinal canal, the intervertebral foramen, and the blood vessel. Finally, the working cannula was removed and the operation was completed.

\section{Statistical analysis}

Data analysis was performed using commercially available software (SPSS version 15.0, SPSS, Inc., Chicago, IL, USA). Continuous variables are presented as the mean \pm standard error of the mean. Qualitative variables are presented as the absolute and relative frequencies. The Wilcoxon rank sum test was used to compare the pre- and post-PKP VAS, ODI, GH and MH scores. Unpaired $t$-tests were used to further assess the differences between groups $\mathrm{A}$ and $\mathrm{B}$ in the VAS, ODI, GH and $\mathrm{MH}$ scores, the volume of PMMA per vertebra, and the operation time. The $\chi^{2}$ or Fisher's exact test was used to compare ratios. $P<0.05$ was considered to indicate a statistically significant difference.

\section{Results}

PKP was successfully completed via a unilateral surgical approach in all patients. The operative time in group A $(0.78 \pm 0.02 \mathrm{hrs})$ was significantly shorter than that in group B $(1.35 \pm 0.05 \mathrm{hrs} ; P<0.001)$. A similar volume of PMMA was injected into each vertebral body in group A $(4.11 \pm 0.07 \mathrm{~mL})$ and group B $(3.92 \pm 0.02 \mathrm{~mL} ; P>0.05)$. The follow-up period was $16 \pm 0.2$ months (range 10 to 36 months) in group A, and $10 \pm 0.9$ months (range 4 to 25 months) in group B.

\section{Treatment efficacy VAS}

The preoperative VAS scores were similar in group A and group B ( $P=0.0585$; Table 3$)$. The effective pain relief rate $(>50 \%$ pain reduction as measured by the VAS) was $82.695 \%$ in group A, and $77.78 \%$ in group B. Both groups experienced significant pain relief after surgery (Figure 1A). 
Table 3 VAS scores during the 6-month follow-up period

\begin{tabular}{|l|l|l|l|l|l|l|}
\hline Group & $\mathbf{2 4}$ h before & I day after & I week after & I month after & 3 months after & $\mathbf{6}$ months after \\
\hline A & $7.67 \pm 0.08$ & $3.60 \pm 0.14$ & $3.41 \pm 0.10$ & $3.69 \pm 0.12$ & $3.91 \pm 0.12$ & $3.80 \pm 0.12$ \\
B & $7.75 \pm 0.09$ & $3.8 I \pm 0.11$ & $3.74 \pm 0.13$ & $3.92 \pm 0.13$ & $3.75 \pm 0.14$ & $3.74 \pm 0.13$ \\
$P^{\mathrm{a}}$ & 0.514 & 0.1846 & 0.044 & 0.216 & 0.373 & 0.734 \\
\hline
\end{tabular}

Note: ${ }^{\text {a }}$-value for comparisons between groups $\mathrm{A}$ and $\mathrm{B}$.
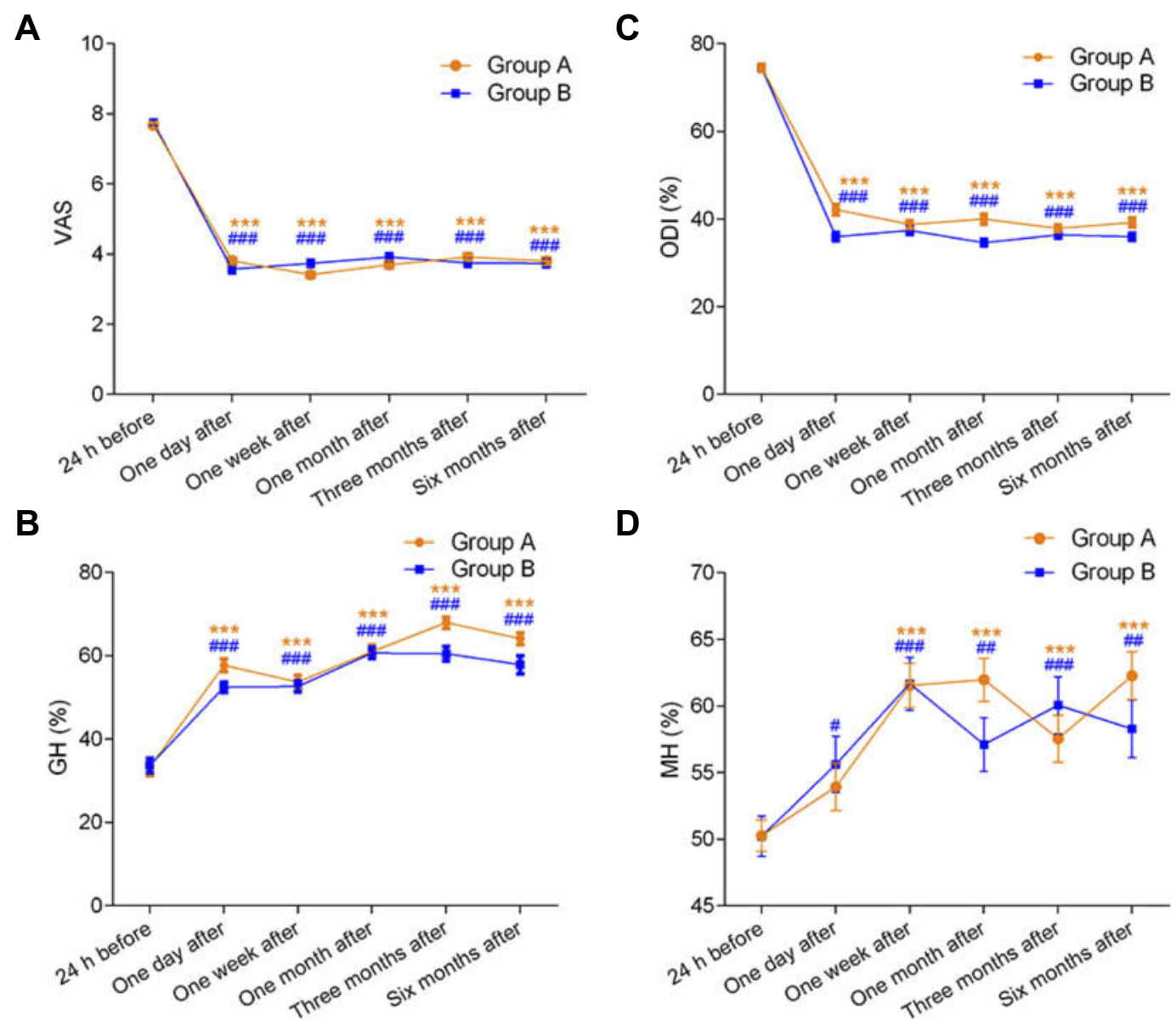

Figure I Pre- and postoperative assessments of pain, function, GH, and MH. (A) VAS, (B) GH score on the Short Form-36 Health Survey, (C) ODI, (D) MH score on the Short Form-36 Health Survey. For the comparison of the pre- and postoperative values within group A, * indicates $P<0.05$, $* *$ indicates $P<0.01$, and $* * *$ indicates $P<0.00 \mathrm{I}$. For the comparison of the pre- and postoperative values within group $B$, \# indicates $P<0.05$, \# indicates $P<0.01$, and \#\# indicates $P<0.001$.

Abbreviations: $\mathrm{GH}$, general health; $\mathrm{MH}$, mental health; ODI, Oswestry Disability Index.

At 1 week after PKP, group A had a significantly lower mean VAS score than group B $(P<0.05$; Table 3$)$.

\section{GH score}

The mean preoperative GH score in group A did not significantly differ from that in group $\mathrm{B}(P=0.881$; Table 4). The GH score in both groups significantly improved after PKP $(P<0.001$; Figure 1B). At 1 day, 3 months, and 6 months after PKP, the mean GH score in group A was significantly greater than that in group B $(P<0.05$; Table 5).

\section{$\mathrm{ODI}$ and $\mathrm{MH}$ score}

The ODI and MH scores were significantly improved after PKP in both groups $(P<0.05$; Figure $1 \mathrm{C}, \mathrm{D})$. There were no significant differences between the two groups in the pre- and postoperative ODI and MH scores ( $P>0.05$; Tables 5 and 6$)$.

\section{Safety}

Postoperative computed tomography showed that local bone cement leakage occurred at 102 levels $(18.09 \%)$, including 35 levels in group A (17.16\%) and 67 levels in group B (18.61\%). The bone cement leaked into the 
Table 4 Oswestry Disability Index during the 6-month follow-up period

\begin{tabular}{|l|l|l|l|l|l|l|}
\hline Group & $\mathbf{2 4} \mathbf{h}$ before & I day after & I week after & I month after & 3 months after & $\mathbf{6}$ months after \\
\hline A & $74.48 \pm 0.81$ & $35.98 \pm 0.92$ & $37.40 \pm 0.84$ & $34.60 \pm 0.74$ & $36.38 \pm 0.68$ & $35.94 \pm 0.78$ \\
B & $74.31 \pm 0.96$ & $38.19 \pm 1.25$ & $35.92 \pm 0.89$ & $34.69 \pm 0.94$ & $35.14 \pm 0.73$ & $35.47 \pm 0.95$ \\
$P^{\text {a }}$ & 0.89 & 0.146 & 0.238 & 0.934 & 0.223 & 0.703 \\
\hline
\end{tabular}

Note: ${ }^{\text {a }}$-value for comparisons between groups $A$ and $B$.

Table 5 General health scores during the 6-month follow-up period

\begin{tabular}{|l|l|l|l|l|l|l|}
\hline Group & $\mathbf{2 4}$ h before & I day after & I week after & I month after & 3 months after & $\mathbf{6}$ months after \\
\hline A & $33.65 \pm 1.68$ & $52.45 \pm 1.20$ & $52.64 \pm 1.33$ & $60.63 \pm 1.34$ & $60.48 \pm 1.71$ & $57.84 \pm 2.19$ \\
B & $33.26 \pm 1.96$ & $57.7 I \pm 1.52$ & $53.68 \pm 1.61$ & $60.97 \pm 1.57$ & $67.92 \pm 1.37$ & $64.10 \pm 1.38$ \\
$P^{\mathrm{a}}$ & 0.881 & 0.007 & 0.62 & 0.867 & 0.002 & 0.031 \\
\hline
\end{tabular}

Note: ${ }^{\text {a }}$-value for comparisons between groups $A$ and $B$.

Table 6 Mental health scores during the 6-month follow-up period

\begin{tabular}{|l|l|l|l|l|l|l|}
\hline Group & $\mathbf{2 4}$ h before & I day after & I week after & I month after & 3 months after & $\mathbf{6}$ months after \\
\hline A & $50.20 \pm 1.18$ & $53.90 \pm 1.78$ & $61.50 \pm 1.67$ & $61.90 \pm 1.63$ & $57.50 \pm 1.77$ & $62.20 \pm 1.80$ \\
B & $50.20 \pm 1.5 \mathrm{I}$ & $55.60 \pm 2.10$ & $61.60 \pm 1.98$ & $57.10 \pm 2.02$ & $60.00 \pm 2.14$ & $58.20 \pm 2.17$ \\
$P^{\mathrm{a}}$ & 0.98 & 0.542 & 0.961 & 0.062 & 0.365 & 0.158 \\
\hline
\end{tabular}

Note: ${ }^{a}$-value for comparisons between groups $A$ and $B$.

adjacent intervertebral disc $(\mathrm{n}=42,7.45 \%)$, peripheral paravertebral tissue $(\mathrm{n}=36,6.38 \%)$, epidural space $(\mathrm{n}=16$, $2.84 \%)$, and needle puncture channel $(\mathrm{n}=8,1.42 \%)$ (Table 7), but no patient had any clinical or neurological symptoms. There were no postoperative complications such as discitis, suture infection, and bone cement syndrome.

\section{Follow-up}

Six months of follow-up was completed for 170 of 176 patients, as six patients in group B died during the followup period. Of these six patients, three died from lung cancer with pneumonia, while three died from cancer-

Table 7 Follow-up results and complications in the two groups

\begin{tabular}{|l|l|l|l|}
\hline & Group A & Group B & P-value \\
\hline Cement leakage & $35(17.16 \%)$ & $67(18.61 \%)$ & 0.168 \\
Adjacent intervertebral disc & $16(7.84 \%)$ & $26(7.22 \%)$ & \\
Peripheral paravertebral tissue & $11(5.39 \%)$ & $25(6.94 \%)$ & \\
Epidural space & $6(2.94 \%)$ & $10(2.78 \%)$ & \\
Puncture channel & $3(1.47 \%)$ & $5(1.39)$ & \\
\hline Follow-up & & & \\
Refracture & $9.8 \%$ & $11.67 \%$ & 0.497 \\
Survival (6 months) & $100 \%$ & $91.67 \%$ & 0.004 \\
\hline
\end{tabular}

associated systemic dyscrasia. Pathological fractures were diagnosed in 20 patients in group A (giving a refracture rate of $9.8 \%$ ), while the refracture rate in group B was $11.67 \%$ (Table 7, $P>0.05$ ).

\section{Discussion}

The absolutely safe number of PKP levels for the treatment of vertebral metastases in one procedure remains controversial. Barr et al reported that singlelevel PKP has better efficacy than multilevel PKP. ${ }^{10}$ Furthermore, Lavanga suggested that PKP should be performed at no more than three levels in a single operation in order to reduce the associated complications and patient discomfort. ${ }^{11}$ However, Zhang et al found no significant difference in the efficacy and safety of vertebroplasty at more than three levels compared with vertebroplasty at less than three levels. ${ }^{12}$ Thus, the safety and efficacy of multilevel PKP for malignant tumors requires further study.

In the present study, the most common primary cancer sites were the lung, breast, and esophagus. Cancers at these sites are more likely to metastasize to the thoracic vertebrae. Thus, thoracic vertebrae were most commonly affected in group B (those with vertebral metastases at four 
or more levels). However, the VAS scores did not significantly differ between group A and group B, despite the weightbearing nature of the thoracic spine and the greater refracture rate in group B. This may be because most patients were lying in bed during the preoperative VAS evaluation, and wore a waist belt postoperatively to avoid spinal overactivity. In addition, most patients were taking analgesics, which would have affected the VAS evaluation.

Back pain was significantly improved after PKP in all patients, with an effective pain relief rate of $80.68 \%$. In the short-term $(<1$ week postoperatively), group B experienced significantly more pain than group A, but there was no significant difference between the groups in long-term pain relief ( $>1$ month postoperatively). Thus, we believe that multilevel PKP is able to achieve the same satisfactory clinical efficacy as PKP at no more than three levels of vertebral metastases. The efficacy of percutaneous osteoplasty in other bone metastases has also been validated. Plancarte et al reported reduced pain intensity, reduced analgesic consumption, and improved quality of life in 80 patients with femoral metastases at 7 and 30 days after percutaneous osteoplasty. ${ }^{13}$ Furthermore, Cazzato et al found that $27.4-68.2 \%$ of patients experienced pain reduction, and $6-71.9 \%$ of patients experienced improvement of limb function. ${ }^{14}$

There are three mechanisms by which PKP may alleviate the pain caused by vertebral metastases. First, bone cement may increase the stability of microfractures, increase bone strength and loadbearing ability, and reduce painful stimulation of peripheral nerves. Second, bone cement may have a certain anti-tumor effect due to the bone cement solidification exothermy and the toxicity of the bone cement monomer. ${ }^{15}$ Third, the direct toxic effects of PMMA monomers on nerve endings and the exothermic response of PMMA polymerization may destroy free nerve endings and thus attenuate pain. ${ }^{16}$

In the long-term ( $>3$ months postoperatively), the mean GH score in group B was significantly worse than that in group A. This may be because the patients in group $\mathrm{B}$ were in the late stage of cancer, and were in worse general condition than those in group A, as shown by the preoperative revised Tokuhashi scores. In addition, we also found that although the mean $\mathrm{MH}$ score was mildly improved postoperatively, it was still low, indicating that most patients still thought that their MH was poor after PKP. Therefore, we believe that the treatment of patients with advanced vertebral metastases requires multidisciplinary treatment involving both psychology and physiology.
In terms of safety, the bone cement leakage rate in vertebral metastases is often higher than that in simple osteoporotic vertebral fractures due to the destruction of the cortical bone of the vertebral body or the tumor-rich blood vessels and blood supply. ${ }^{17}$ Thus, for patients with osteolytic vertebral metastases, we often recommend PKP rather than percutaneous vertebroplasty (PVP). Compared with PVP, PKP has a lower risk of cement leakage, as the balloon expansion of the vertebral body results in relatively reduced pressure in the vertebral body when the bone cement is injected. In the present study, the average rates of cement leakage per vertebral body in groups A and B were $17.16 \%$ and $18.61 \%$, respectively, which are indeed lower than the previously reported cement leakage rate at each PVP level of $25.1 \% .^{12}$ Furthermore, in the present study, most of the cement leaked into the adjacent intervertebral disc, and so did not cause neurological symptoms. Therefore, we consider PKP a better option for osteolytic vertebral metastases than PVP. However, the rate of refracture after PKP is reportedly higher than that after PVP. This may be because the bone cement cannot completely fill the cavity expanded by the balloon, resulting in an increase in the long-term refracture rate. In our study, the overall refracture rate was $10.99 \%$, which is lower than that previously reported after PVP (15.2\%). ${ }^{4}$ Furthermore, group B did not have a significantly higher refracture rate than group A. In our experience, it is important to keep the pressure of the gauge pressurizer below 20 ATM and to not excessively expand the vertebral body via the balloon. In addition, the entire cavity must be filled with sufficient PMMA in accordance with the pressure and volume calculated when expanding the vertebral body. Generally, each thoracic vertebra requires the injection of 3-4 mL of PMMA, while each lumbar vertebrae requires $4-5 \mathrm{~mL}$ of PMMA.

During the follow-up period, three patients in group $B$ died from lung cancer with pneumonia at 4 months after PKP, and three patients in group B died from cancer-associated systemic dyscrasia. It should be emphasized that PKP is only a local palliative analgesic treatment for patients with multiple vertebral metastases. Prolonged patient survival requires systemic treatments such as radiation therapy, chemotherapy, and immunotherapy. Therefore, patients should continue to undergo treatments for primary tumors and systemic support after discharge to prolong survival. 


\section{Limitations}

The present study had some limitations. First, the retrospective study design means that selection bias was unavoidable. Second, besides PKP, other treatments such as interventional tumor resection and ablation may provide relatively low complication and high efficacy rates, ${ }^{18-20}$ and these techniques should be investigated in future studies. Third, the disease stage and adjuvant treatments are important confounders that may cause pain; however, these variables were not assessed in the present study. Fourth, the VAS used in the present study is not specific for the evaluation of pain caused by vertebral metastases, as other metastatic sites may also have been causing pain.

\section{Conclusion}

Multilevel PKP for osteolytic vertebral metastases results in safe and effective pain relief, and improvements in spinal mobility and GH. However, patients with multilevel PKP had slightly worse pain relief in the short-term (less than 1 week postoperatively) and improvement of $\mathrm{GH}$ in the long-term (more than 3 months postoperatively) than patients with PKP at less than three levels.

\section{Acknowledgments}

No funds were received in support of this work. No benefits in any form have been or will be received from a commercial party related directly or indirectly to the subject of this manuscript.

\section{Disclosure}

The authors declare that there are no conflict of interests regarding the publication of this paper.

\section{Ethical approval}

The study was conducted in accordance with the ethical standards of the Medical Ethics Committee of Beijing Friendship Hospital, Capital Medical University and with the 1975 Declaration of Helsinki, as revised in 2000.

\section{References}

1. Bouras T, Zairi F, Arikat A, et al. Decision making for the surgical treatment of vertebral metastases among patients with short predicted survival. World Neurosurg. 2018;111:573-580. doi:10.1016/j. wneu.2017.12.107

2. Chew C, O'Dwyer PJ, Edwards R. Health service cost associated with percutaneous vertebroplasty in patients with spinal metastases. Clin Radiol. 2013;68(8):776-779.
3. Hariri O, Takayanagi A, Miulli DE, et al. Minimally invasive surgical techniques for management of painful metastatic and primary spinal tumors. Cureus. 2017;9(3):1114.

4. Kircelli A, Coven I. Percutaneous balloon kyphoplasty vertebral augmentation for compression fracture due to vertebral metastasis: a 12-month retrospective clinical study in 72 patients. Med Sci Monit. 2018;24:2142-2148.

5. Zhang HT, Chen GD, Yang HL, et al. Percutaneous kyphoplasty in the treatment of osteoblastic-related spinal metastases. Clin Spine Surg. 2017;30(2):80-84.

6. Yaltirik K, Ashour AM, Reis CR, et al. Vertebral augmentation by kyphoplasty and vertebroplasty: 8 years experience outcomes and complications. J Craniovertebr Junction Spine. 2016;7 (3):153-160.

7. Ziad AA, Mahmoud HH, Mohammad DF, et al. Comparison if the addition of multilevel vertebral augmentation to conventional therapy will improve the outcome of patients with multiple myeloma. Scoliosis Spinal Disord. 2016;11(1):47.

8. La MGA, Sala F, Callea G, et al. Efficacy of unipedicular baloon kyphoplasty for treatment of multiple myeloma vertebral lesions. Asian Spine J. 2011;5(3):162-168.

9. Katonis P, Hadjipavlou A, Souvatzis X, et al. Respiratory effects, hemodynamic changes and cement leakage during multilevel cement balloon kyphoplasty. Eur Spine J. 2012;21(9):18601866.

10. Barr JD, Barr MS, Lemley TJ, et al. Percutaneous vertebroplasty for pain relief and spinal stabilization. Spine (Phila Pa 1976). 2000;25 (8):923-928.

11. Lavanga A, Guarnieri GMM. Medical therapy and multilevel vertebroplasty in osteoporosis: when and why. Neuroradiol J. 2010;23 (2):244-248. doi:10.1177/197140091002300215

12. Zhang JJ, Zhou Y, Hu HY, et al. Safety and efficacy of multilevel vertebroplasty for painful osteolytic spinal metastases: a single-centre experience. Eur Radiol. 2017;27(8):3436-3442. doi:10.1007/s00330016-4683-x

13. Plancarte, R., Guajardo, J., Meneses-Garcia, A. et al. Clinical benefits of femoroplasty: a nonsurgical alternative for the management of femoral metastases. Pain Physician. 2014;17(3):227-234.

14. Cazzato R, Palussière J, Buy X, et al. Percutaneous long bone cementoplasty for palliation of malignant lesions of the limbs: a systematic review. Cardiovasc Intervent Radiol. 2015;38 (6):1563-1572. doi:10.1007/s00270-015-1082-7

15. Baroud G, Samara M, Steffen T. Influence of mixing method on the cement temperature-mixing time history and doughing time of three acrylic cements for vertebroplasty. J Biomed Mater Res B Appl Biomater. 2004;68(1):112-116. doi:10.1002/jbm.b.20009

16. Belkoff SM, Molloy S. Temperature measurement during polymerization of polymethymethacrylate cement used for vertebroplasty. Spine (Phila Pa 1976). 2003;28(14):1555-1559.

17. Sun H, Yang Z, Xu Y, et al. Safety of percutaneous vertebroplasty for the treatment of metastatic spinal tumors in patients with posterior wall defects. Eur Spine J. 2015;24(8):1768-1777. doi:10.1007/s00586-0153810-8

18. Jakobs TF, Trumm C, Reiser M, et al. Percutaneous vertebroplasty in tumoral osteolysis. Eur Radiol. 2007;17(8):2166-2175. doi:10.1007/ s00330-007-0581-6

19. Clarencon F, Jean B, Pham HP, et al. Value of percutaneous radiofrequency ablation with or without percutaneous vertebroplasty for pain relief and functional recovery in painful bone metastases. Skeletal Radiol. 2013;42(1):25-36.

20. Li Y, Gu YF, Sun ZK, et al. Comparison of percutaneous vertebroplasty with and without interventional tumour removal for malignant vertebral compression fractures with symptoms of neurological compression. Eur Radiol. 2013;23(10):2754-2763. 


\section{Publish your work in this journal}

The Journal of Pain Research is an international, peer reviewed, open access, online journal that welcomes laboratory and clinical findings in the fields of pain research and the prevention and management of pain. Original research, reviews, symposium reports, hypothesis formation and commentaries are all considered for publication. The manuscript management system is completely online and includes a very quick and fair peer-review system, which is all easy to use. Visit http:// www.dovepress.com/testimonials.php to read real quotes from published authors. 